\title{
L'Europe et le monde germanique (Époque moderne et contemporaine)
}

Jacques Le Rider

\section{OpenEdition}

\section{Journals}

Édition électronique

URL : https://journals.openedition.org/ashp/1038

DOI : $10.4000 /$ ashp.1038

ISSN : 1969-6310

\section{Éditeur}

Publications de l'École Pratique des Hautes Études

\section{Édition imprimée}

Date de publication : 2 février 2011

Pagination : 290-291

ISSN : 0766-0677

\section{Référence électronique}

Jacques Le Rider, «L'Europe et le monde germanique (Époque moderne et contemporaine) », Annuaire de l'École pratique des hautes études (EPHE), Section des sciences historiques et philologiques [En ligne],

141 | 2011, mis en ligne le 24 février 2011, consulté le 06 juillet 2021. URL : http:// journals.openedition.org/ashp/1038; DOI : https://doi.org/10.4000/ashp.1038 


\title{
L'EUROPE ET LE MONDE GERMANIQUE (ÉPOQUE MODERNE ET CONTEMPORAINE)
}

\author{
Directeur d'études : M. Jacques LE RIDER
}

Programme de l'année 2008-2009: De la crise de l'assimilation au scepticisme et à l'athéisme. Fritz Mauthner (1894-1923).

Né en 1849 dans une famille juive de culture allemande, Mauthner passa son enfance dans la petite ville de Horzitz (Horice), voisine de Königgrätz, en Bohême, puis, de 1855 à 1876, sa jeunesse à Prague. L'expérience de la guerre des langues le conduit à réfuter, dans ses mémoires intitulés Une jeunesse pragoise, publiés en 1918, ce que Claudio Magris appelle « le mythe habsbourgeois » de la coexistence harmonieuse des nationalités en Autriche-Hongrie. Après avoir quitté Prague en 1876 pour faire carrière à Berlin, il fut jusqu'à 1905 un des journalistes les plus connus de Berlin, critique littéraire et théâtral, mais aussi romancier. Son roman Der neue Ahasver (Le Nouveau Juif errant), publié en 1881, prend position dans la controverse qui opposait les antisémites, rassemblés autour de l'historien Treitschke, et les intellectuels anti-antisémites, parmi lesquels Mommsen était le plus connu.

Les Contributions à une critique du langage (Beiträge zu einer Kritik der Sprache) de Mauthner, publiées en 1901-1902, eurent un retentissement considérable. Dans les trois tomes de cet essai monumental (plus de deux mille pages), le scepticisme linguistique prend les dimensions d'un système que l'on peut situer dans le contexte de la Lettre de Lord Chandos de Hugo von Hofmannsthal ou des satires de Karl Kraus dénonçant la dépravation de la langue dans la presse contemporaine.

Radicalisant la critique schopenhauerienne et nietzschéenne du langage, mais aussi la tradition de l'empirisme anglais, de Locke à Hume, Mauthner soutient que penser, ce n'est que parler. L'intellect se réduit, selon lui, à une activité linguistique. Le sujet parlant est rempli de sensations et de souvenirs de sensations que le langage sert à mettre dans un semblant d'ordre, construisant une vision du monde anthropomorphique différente de ce que Mauthner appelle Wirklichkeitswelt. La communication est une illusion : si tous les objets sont des objets internes, c'est-à-dire des sensations, il est illusoire de penser que toutes les subjectivités perçoivent les mêmes objets. Mais la langue produit un «effet de communication » qui pousse les locuteurs, lorsqu'ils utilisent les mêmes mots, à croire qu'ils parlent des mêmes choses et que les individus entre en dialogue, alors que les mots, en dehors des actes de langage de la vie quotidienne, les séparent et les isolent, car ils composent, selon Mauthner, un langage privé propre à chaque subjectivité.

Chez Mauthner, il s'agit de bien plus que d'une critique du langage : c'est une véritable haine des mots qui s'exprime dans son réquisitoire fleuve contre la langue qu'il considère comme un système trompeur de signes arbitraires emprisonnant l'individu, l'induisant en erreur et ne le conduisant à aucune vérité. Les mots sont coupés des choses. Le langage est une interprétation anthropomorphique du réel, une vision 
du monde. La connaissance de la réalité qui s'exprime dans le langage conceptuel est illusoire.

Ce scepticisme radical conduit au nihilisme. Faisant table rase de ce qui ancre la culture dans le logos, de ce qu'il appelle la «logocratie », Mauthner met à part le langage poétique : ayant affirmé que les mots ne sauraient servir à la connaissance de la réalité, il définit la littérature comme Sprachkunst. C'est pourquoi le traité de Mauthner (qui était lui-même, comme poète et comme romancier, tout le contraire d'un écrivain d'avant-garde) est considéré comme un texte-source de la modernité : de son contemporain Christian Morgenstern à Hugo Ball, dans le manifeste dada de juillet 1916, et au groupe de Vienne (Oswald Wiener), nombre d'auteurs expérimentaux se réfèrent à Fritz Mauthner. D'autres prennent son contrepied : ainsi Walter Benjamin, qui s'était fait offrir un ouvrage de Mauthner pour son dix-huitième anniversaire, rejettera les perspectives du nihilisme linguistique, exprimant dans son essai sur le langage de 1916 la nostalgie utopique d'une langue ayant retrouvé son pouvoir « magique » de réconcilier l'individu avec lui-même, avec la nature et avec la société. Jorge Luis Borges appréciait beaucoup son Dictionnaire de la philosophie et James Joyce, en 1932, recommandait à Samuel Beckett la lecture des Contributions à une critique du langage de Mauthner. Dans Réelles présences, George Steiner insiste sur l'importance des Contributions à une critique du langage.

Le pouvoir des mots est pour Mauthner, au contraire, une magie noire dont il veut désensorceller ses contemporains. Son scepticisme linguistique aboutit chez Mauthner à une «mystique sans Dieu », dont Gustav Landauer, figure de proue du socialisme anarchiste, redécouvreur de Maître Eckhart, donne la formule dans Scepticisme et mystique. Essais dans le prolongement de Mauthner (Skepsis und Mystik. Versuche im Anschluss an Mauthner, 1903). La «mystique sans Dieu » de Mauthner fascinera aussi Martin Buber : c'est pour la collection dirigée par Martin Buber que Mauthner écrira le résumé synthétique le plus abouti de sa pensée sous le titre de Die Sprache, Le Langage, publié en 1907. Dans le Tractatus logico-philosophicus, Ludwig Wittgenstein définira sa propre position en se démarquant de Mauthner (4.0031: « Toute philosophie est "critique du langage". (Mais non, il est vrai, dans le sens de Mauthner.) ») : il reste que les textes de Mauthner permettent de situer certains traits du Tractatus dans leur contexte historique, particulièrement la tendance «mystique » qui s'exprime dans la proposition 6.522 : «En tout cas, il y a de l'indicible. Il se montre; c'est cela le mystique. »

De 1905 à 1909, après avoir rompu avec sa carrière journalistique et littéraire, Mauthner se retire à Fribourg-en-Brisgau, où il suit des enseignements dans plusieurs Facultés, tout en poursuivant ses recherches théologiques, de philosophiques et linguistiques. Établi à Meersburg à partir de 1911, il se consacre à son Dictionnaire de la philosophie (Wörterbuch der Philosophie: Neue Beiträge zu einer Kritik der Sprache, première édition vol. 1 et 2, 1910-1911; deuxième édition en trois volumes, 19231924 ) et à son dernier ouvrage de grande envergure, L'Athéisme et son histoire en Occident (Der Atheismus und seine Geschichte im Abendlande), publié entre 1920 et 1923. Ce dernier ouvrage tire les conséquences de l'aphorisme de Nietzsche dans Le crépuscule des idoles: « Je crains bien que nous ne nous débarrassions jamais de Dieu, puisque nous croyons encore à la grammaire. » 